\title{
Anomalous thermal behaviour and diffuse scattering in cadmium cyanide
}

Chloe Simone Coates ${ }^{1}$, Mia Baise ${ }^{2}$, Arkadiy Simonov ${ }^{1}$, Ben Slater ${ }^{2}$, Andrew Goodwin ${ }^{1}$

${ }^{1}$ Department Of Chemistry, University Of Oxford, Oxford, United Kingdom, ${ }^{2}$ Department of Chemistry, University College London, London, United Kingdom

E-mail: chloe.coates@chem.ox.ac.uk

Whilst traditionally crystallography has been applied uniquely to ordered materials, there is burgeoning interest in the analysis of disordered materials and how they might be exploited for their functionality. $\mathrm{Cd}(\mathrm{CN}) 2$ has attracted considerable interest for its very strong negative thermal expansion (NTE). It adopts the interpenetrated diamondoid structure of cubic ice-VII and favours the 'two-in-two-out' cyanide configuration that governs proton disorder in ice, and gives rise to residual entropy of ice at $0 \mathrm{~K}$ and magnetic monopoles and loop states in spin-ices.

The correlated disorder in $\mathrm{Cd}(\mathrm{CN}) 2$ that arises from the static orientational disorder of the cyanides, as well as concomitant displacements of the $\mathrm{Cd}$ centres, has its signature in the highly structured diffuse scattering, which (unusually) increases in intensity down to a phase transition at $150 \mathrm{~K}$ which sees the emergence of multiple polymorphs. This very complex and heretofore unsolved phase transition in crucial in understanding the vibrational behaviour that governs the NTE.

Here I will present this system's anomalous responses to temperature (NTE) on the one hand, and x-ray exposure on the other hand, as determined using powder x-ray diffraction. We characterise its unusual behaviour at low temperature; present supporting evidence from DFT and reveal the structure of the low temperature polymorph, which represents a new topology for this system, one that is reminiscent of the phase transition of ice-VII-ice-VIII. We explore further the effect of temperature on a range of framework topologies, accessed via cadmium cyanide clathrates (Cd(CN)2.G, G=guest), in order to establish a clear relationship between anomalous physical properties and the degree of correlated disorder.

Preliminary high-pressure studies show compressibility that is an order of magnitude greater than that predicted from DFT as well as phase transitions and the unusual property of pressure induced softening, as seen in isostructural $\mathrm{Zn}(\mathrm{CN}) 2$.

This is an ideal system to probe the relationship between disorder and material properties, and give an indication as to how we might tune the structure, disorder and, in turn, functionality of framework materials.

[1] A. L. Goodwin et al., Phys. Rev. B 71, 140301(R) (2005)

[2] S-I Nishikiori et al., Can J Chem 68, 2770 (1990)

[3] V. E. Fairbank et al., Phys Rev B, 86, 104113 (2012)

Keywords: negative thermal expansion, disorder, diffuse scattering 